\title{
AN EDUCATIONAL PLANT BASED ON THE QUADRUPLE-TANK PROCESS
}

\author{
I. Alvarado ${ }^{1}$ D. Limon ${ }^{1}$ W. García-Gabín ${ }^{2}$ T. Alamo ${ }^{1}$ \\ E.F. Camacho ${ }^{1}$
}

\begin{abstract}
This paper presents an experimental tank system developed at the University of Seville for process control education. This plant is based on the well known quadrupletank process and some modifications have been done in order to obtain a wide range of applications. The quadruple tank process is a multivariable laboratory plant of interconnected tanks that can be easily configured to exhibit the effect of multivariable zero (minimum and non-minimum phase) on the system behavior, as well as the effect of non linear dynamics, saturation, constraints, etc.

In the real plant implementation, the original structure of the process has been modified to offer a wide variety of uses for both educational and research purposes.Thus, different plants can be configured such as one single tank, two or three cascaded tanks, a mixture process and hybrid dynamics. Moreover the dynamics parameters of each tank can be set up by tuning the cross-section of the outlet hole of the tank. Furthermore, the real plant has been implemented using industrial instrumentation and a PLC for the low level control. Supervision and control of the plant is carried out in a computer by means of OPC (Ole for Process Control) which allows one to connect the plant with a wide range of control programs such as LabView, Matlab or industrial SCADA.
\end{abstract}

Keywords:

Process control education, laboratory plant design, multivariable zeros, OPC

\section{INTRODUCTION}

One of the difficulties encountered in control education consists of providing a theoretical foundation maintaining the practicality. To this aim, experimental labs provide a powerful tool to fill this gap. An experimental lab should be designed to show interesting and industrially relevant control problems which require not too skilled control solutions and real tools, such as instrumentation, control programs, etc.

The quadruple tank process has proved to be a very interesting system for control education in ad-

\footnotetext{
1 Dpto de Ingeniería de Sistemas y Automática, Escuela Superior de Ingenieros, Universidad de Sevilla. Avda/ Camino de los Descubrimientos s/n. 41092, Sevilla (Spain). \{alvarado, limon, al amo, eduardo\}@cartuja.us.es 2 University of Los Andes, Venezuela.

winstong@ula.ve

3 The authors gracefully acknowledges MCYT (Spain) contract DPI 2004-07444 for funding this work.
}

vanced control courses as well as in research courses (Johansson, 2000; Johansson et al., 1999; Rusli et al., 2004; Long et al., 2005). The main property of this process is that it is appropriate to illustrate the importance of multivariable zeros since these can be located at the right and the left half plane. Furthermore, there exist other insteresting properties, such as the coupled nature of the plant, the measurable states, the nonlinear behavior, for instance.

To this aim, a laboratory plant based on the quadruple tank process has been designed and developed at University of Seville. This plant is used for both educational and research purposes. The objective of the design has been to provide flexibility to the plant. Thus, the plant can be easily configured to obtain different processes and the control system has been implemented to allow us to control from the PLC, from an external device or from a computer by means of an open and standard protocol OPC (OLE for Process 


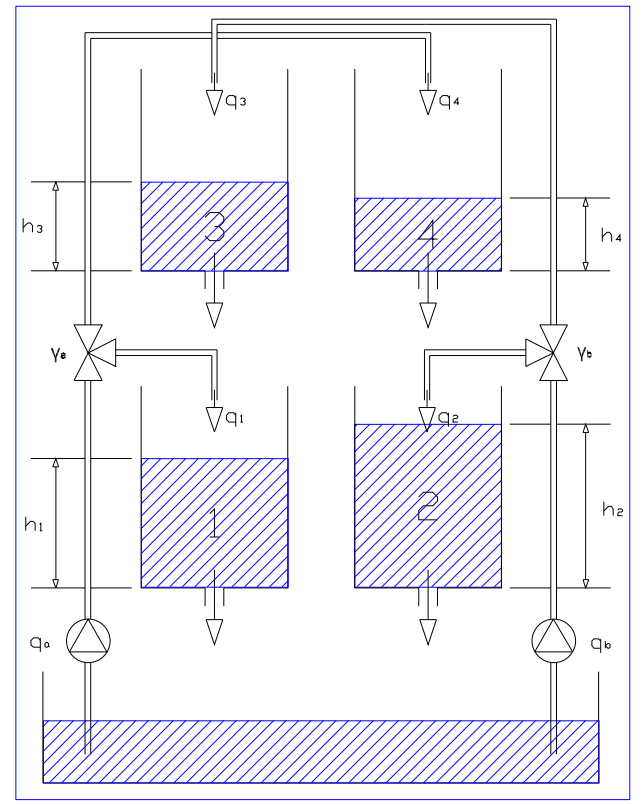

Fig. 1. The Quadruple Tank Process scheme.

Control). Thus, any control software with OPC connectivity (such as MATLAB, LAbView or commercial SCADAs) can be used to control the plant.

The paper is organized as follows: first, the quadruple tank process is presented in section 2 and the implemented plant is described in the following section. In section 4 the instrumentation used in the plant is presented and in section 5 the control structure is demonstrated. The paper draws to a close with some conclusions.

\section{THE QUADRUPLE TANK PROCESS}

This process is a laboratory plant proposed in (Johansson, 2000) aimed to show the effect of non-minimum zeros of a multivariable system. The original plant consists of four interconnected tanks as shown in Fig 1. The inputs are the voltages of the two pumps and the outputs are the water levels in the lower two tanks.

The model of the system (Johansson, 2000) is derived from first principles as follows

$$
\begin{aligned}
\frac{d h_{1}}{d t} & =-\frac{a_{1}}{A_{1}} \sqrt{2 g h_{1}}+\frac{a_{3}}{A_{1}} \sqrt{2 g h_{3}}+\frac{\gamma_{1}}{A_{1}} q_{a} \\
\frac{d h_{2}}{d t} & =-\frac{a_{2}}{A_{2}} \sqrt{2 g h_{2}}+\frac{a_{4}}{A_{2}} \sqrt{2 g h_{4}}+\frac{\gamma_{2}}{A_{2}} q_{b} \\
\frac{d h_{3}}{d t} & =-\frac{a_{3}}{A_{3}} \sqrt{2 g h_{3}}+\frac{\left(1-\gamma_{2}\right)}{A_{3}} q_{b} \\
\frac{d h_{4}}{d t} & =-\frac{a_{4}}{A_{4}} \sqrt{2 g h_{4}}+\frac{\left(1-\gamma_{1}\right)}{A_{4}} q_{a}
\end{aligned}
$$

where the parameters of the plant are:

\begin{tabular}{|c|c|l|}
\hline State Variables & Unit & Concept \\
\hline \hline$A_{i}$ & $\mathrm{~cm}^{2}$ & Cross-section of tank i \\
$a_{i}$ & $\mathrm{~cm}^{2}$ & Cross-section of the outlet hole \\
$h_{i}$ & $m$ & Water level of the Tank i \\
$q_{a}, q_{b}$ & $\mathrm{~m}^{3} / h$ & Flow over the pumps \\
$g$ & $\mathrm{~m} / \mathrm{s}^{2}$ & The acceleration of gravity \\
$q_{i}$ & $\mathrm{~m}^{3} / h$ & Flow over the each tank \\
$\gamma_{i}$ & & Parameters of the three-way valves \\
\hline
\end{tabular}

Linearizing the model in an operating point given by $h_{i}^{0}$ and defining the variables $x_{i}=h_{i}-h_{i}^{o}$ and $u_{j}=q_{j}-$ $q_{j}^{o}$ where $j=a, b$ and $i=1, \cdots, 4$ we have that:

$$
\begin{aligned}
\frac{d x}{d t} & =\left[\begin{array}{cccc}
\frac{-1}{T_{1}} & 0 & \frac{A_{3}}{A_{1} T_{3}} & 0 \\
0 & \frac{-1}{T_{2}} & 0 & \frac{A_{4}}{A_{2} T_{4}} \\
0 & 0 & \frac{-1}{T_{3}} & 0 \\
0 & 0 & 0 & \frac{-1}{T_{4}}
\end{array}\right] x+\left[\begin{array}{cc}
\frac{\gamma_{1}}{A_{1}} & 0 \\
0 & \frac{\gamma_{2}}{A_{2}} \\
0 & \frac{\left(1-\gamma_{2}\right)}{A_{3}} \\
\frac{\left(1-\gamma_{1}\right)}{A_{4}} & 0
\end{array}\right] u \\
y & =\left[\begin{array}{llll}
1 & 0 & 0 & 0 \\
0 & 1 & 0 & 0
\end{array}\right] x
\end{aligned}
$$

where $T_{i}=\frac{A_{i}}{a_{i}} \sqrt{\frac{2 h_{i}^{0}}{g}} \geq 0, i=1, \cdots, 4$, are the time constants of each tank.

The system is open loop stable with two multivariable zeros. The nature of these zeros is determined by the parameters $\gamma_{1}$ and $\gamma_{2}$ as follows

- If $0 \leq \gamma_{1}+\gamma_{2}<1$ the system has Right Half Plane transmission Zeros (RHPZ).

- If $1<\gamma_{1}+\gamma_{2} \leq 2$ has Left Half Plane transmission Zeros (LHPZ)

It is worth remarking that the sign of the real part of the zeros does not depend on the operating point.

In addition to this remarkable property, the plant possesses another interesting features that make the plant appropriate to be used for both educational and research purposes. These are the following:

(1) The linearized model of the quadruple-tank process has a multivariable zero, which can be located in either the left or the right half-plane by simply changing a couple of valves.

(2) All the states are measurable.

(3) The outputs are strongly coupled.

(4) The system is nonlinear.

(5) The states and inputs of the plant are constrained.

(6) The plant is easyly harmlessly handled.

Thus this plant can be used to show very interesting control problems. Among these problems, the following ones can be highlighted:

- Control of multivariable systems

- Control of systems with RHPZ and limits of performance.

- Robust control.

- State estimation. 
- Tracking of constant references.

- Control under saturating actions.

- Control of systems subject to constraints.

The design and implementation of the plant has been carried out in such a way that the potential educational interest is maximized. This is detailed in the following section.

\section{IMPLEMENTATION OF THE LABORATORY PLANT}

One of the main objectives in the implementation of the quadruple tank process has been to provide flexibility to the plant in the following aspects:

- Capability to setup different processes in the same plant.

- Capability to tune some parameters which allows us to configure the plant dynamics.

- Wide range of operating points.

Thus, the plant layout has been designed to meet these specifications (see figure 8). The designed plant differs from the quadruple tank process proposed in (Johansson, 2000) in the following items:

- The three-way valve has been replaced by two control valves controlling the flow of the pipes. This allows us to fix a desired flow ratio between the two pipes (that is, the parameter $\gamma_{i}$ ) and hence obtain an ideal three-way valve.

Moreover, given that all the flows of the inlet pipes of the tanks are controlled, different processes can be configured.

- Extra pipes, manipulable valves and tank interconnections have been added to setup the different processes. These will be shown in the following section.

- A manipulated valve with a position display has been placed in the outlets of the tanks in order to manipulate the cross-section of the outlet hole $a_{i}$. This allows us to configure the dynamics of each tank of the process.

- The tanks are transparent, with a rectangular cross section and can be easily removed. This allows one to put in some ad hoc element to change the cross section, and hence change the dynamics of the tank.

Another relevant aspect of the plant is that it has been implemented using industrial measurement devices and industrial control valves, which provides a realistic framework to test controllers. These, together with the low-level control system, will be shown later on. A photograph of the implemented plant can be seen in figure 2. As it can be seen, this plant is larger that the typical scaled lab plants (Rusli et al., 2004; Johansson et al., 1999) (the total height is $3.5 \mathrm{~m}$ and the tanks are $1.3 \mathrm{~m}$ tall).

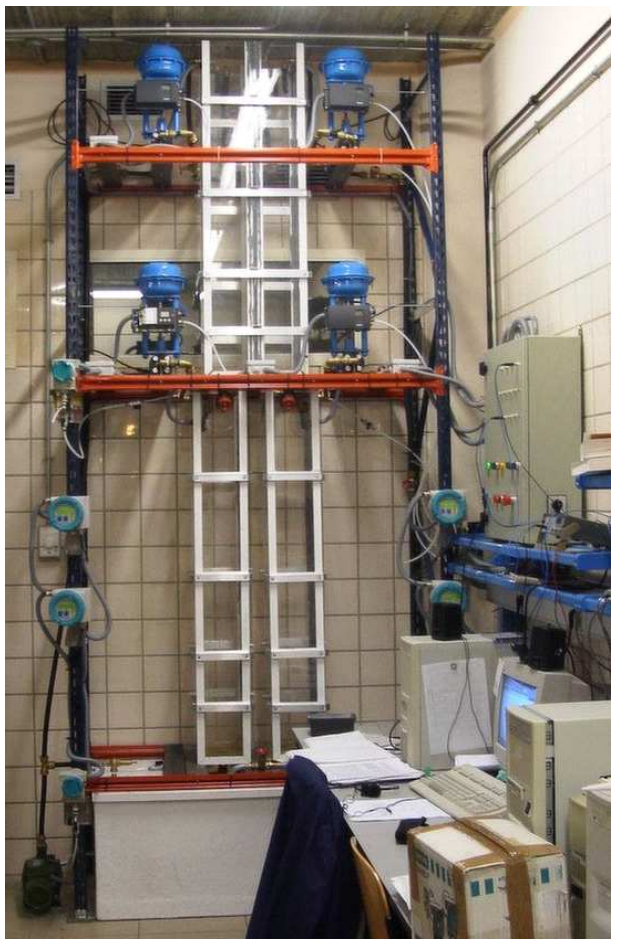

Fig. 2. The implemented laboratory plant.

\subsection{Setting up the plant}

One of the main pros of the designed plant is the capability to configure different processes and dynamics. In the appendix some of the possible configurations are shown (see figure 9). These can be achieved by opening and closing some of the manipulable valves. See that the location of control valves in the tank inlets allows us to obtain a larger number of possible configurations.

The most immediate processes that can be configured are two, three and four cascaded tanks; it is also possible to configure a mixture process, where the reservoir is split in two parts, containing the two products to mix; these are mixed in one of the upper tanks and the mixture is stored in the bottom tanks, connected to enlarge the capacity. Another interesting process is a simple hybrid system. This can be obtained by connecting the two lower tanks at a certain height. Thus, the dynamic changes when some of the two levels are above this height.

Dynamics of the plant can be set up by adjusting the cross-section of the outlets of the tanks. See that this parameter determines the constant time of the tanks and also the water level at a given operating point. Thus these parameters can be fixed with two objectives: change the time response of the system or change the range of variation of the level. The dynamics can also be tuned by changing the cross section of the tank, putting an element to change the variation of the volume with the level. This allows us to enlarge the degree of nonlinearity of the plant or even obtain an hybrid system. 
In the following section, some details of the instrumentation are presented.

\section{INSTRUMENTATION OF THE PLANT.}

The designed plant requires at least some devices to measure the water level in the tanks and control valves at each inlet of the tank. Moreover, in order to ensure the flow of water in each inlet, a flow sensor has been added. The layout of the instruments in the plant and their wiring diagram is shown in figure 3.

All the instruments used in the plant are standard devices used in the process industry. The level of the tanks is measured by pressure sensor and the flows of the inlets by magnetic flow-meters. A pneumatic control valve with positioner has been chosen to manipulate the flows of the inlets. The main reason why industrial instrumentation has been used is to provide a realistic benchmark to test controllers and teach the students to use, configure and work with such devices.

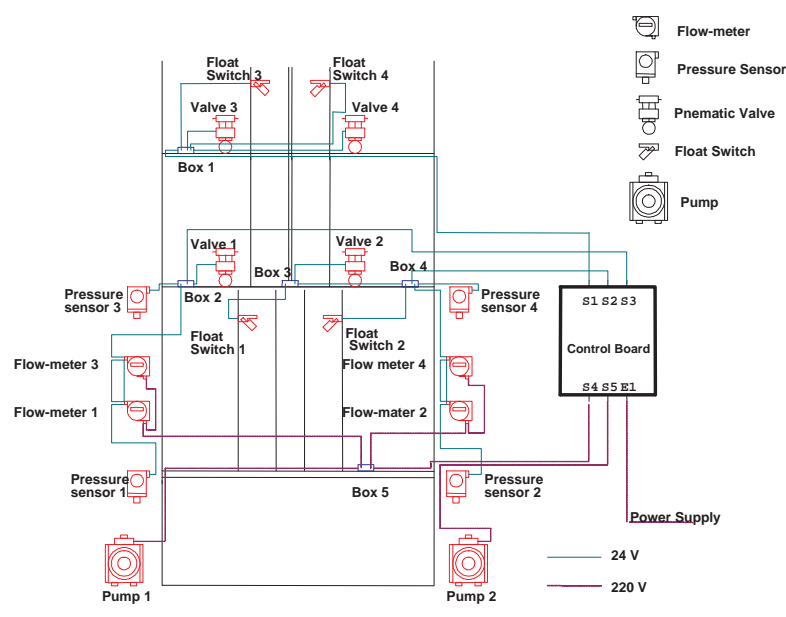

Fig. 3. Instrumentation and wiring of the plant

The measurement provided by the sensors as well as the aperture of the valves are electrical signals (4-20 mA current loop). The wiring of this signals allows one to connect them to a Programmable Logic Controller (PLC) located in the control board or to connect them to an external device by means of plugs located in the panel of the control board (see for instance the wiring of the pressure sensor in the figure 4 , where this is illustrated).

In order to avoid overflows of the tanks, a float is installed at the top of each tank and the on/off signal is wired to the control board where an emergency stop of the plant is carried out closing the valves and stopping the pumps.

The PLC used as data acquisition device allows one to use different control structures, which are presented in the following section.

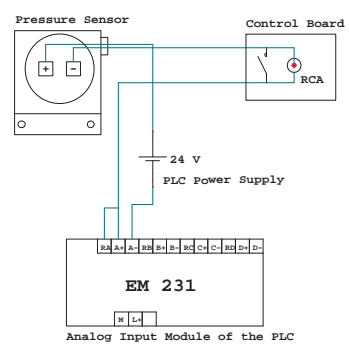

Fig. 4. Wiring of the pressure sensor.

\section{THE CONTROL STRUCTURE OF THE PLANT.}

The control objective of the plant depends on the chosen configuration although this is basically to regulate the levels of the tank. The plant is designed in such a way that allows one to choose different control structures to carry out the control task. This control task is typically carried out in two structures:

Cascaded Control: the control is divided in two levels (or loops): a lower level (inner loop) aimed to control the flow of each inlet and a higher level (outer loop) where the the levels are controlled.

Direct Control: the levels are controlled manipulating directly the apertures of the valves.

On the other hand, the control law can be implemented in the PLC or by means of an external device given that all the signals (measurements and control actions) are accessible in the control board. The external control is interesting from an educational and practical point of view since this allows us to control the plant by means of standard low level controllers, such as industrial PIDs, or using different PLCs or data acquisition systems.

The PLC located in the control board can be used to implement (simple) control laws, such as the low level controllers or to be connected to a computer, allowing us to control the plant by means of an application running on the laptop. In figure 5 it is shown the diagram of the cascaded control structure where the low level PIDs are implemented in the PLC and the high level controller is implemented in the computer.

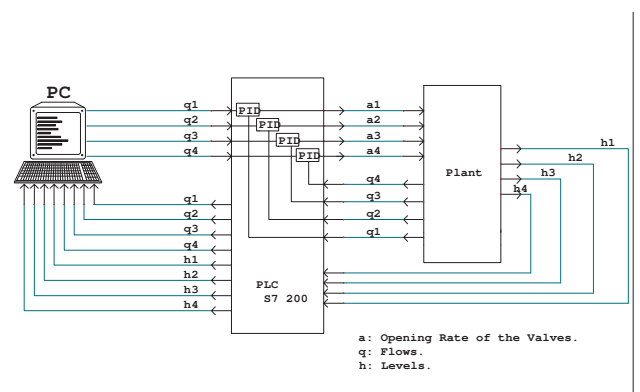

Fig. 5. The computer-based cascaded control structure

All the process variables are periodically sampled and stored in the PLC RAM in a real time data base. These variables can be accessed from the computer thanks 


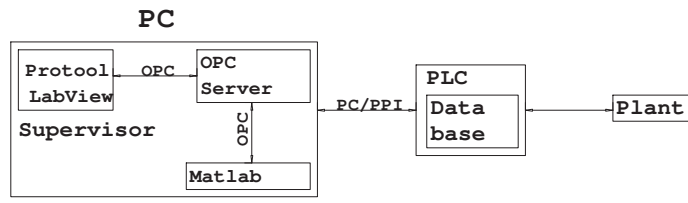

Fig. 6. Computer controlled system by means of OPC to the existing connection with the computer. This connection is an standard serial RS-232 under an open and simple character-based protocol fast enough to control the plant and that can be easily implemented.

Thus, the most simple way to program an application to control the plant is to code the protocol and sampling the state by reading the values from the data base in the PLC and manipulating the plant by writing the control actions in this data base. We have used this approach to develop a LabView based application to monitorize and control the plant.

In order to enhance the connectivity of the process we have chosen an open and free protocol that can be easily found in control packets and SCADAs: the OPC (OLE for Process Control). Thus, an OPC server developed by Kepware Inc. has been installed. This software reads the variables from the data base of the PLC and builds a real time data base in the PC. These variables can be used (read and/or write) by other applications by means of the OPC protocol in a serverclient architecture (see figure 6. Control software such as MATLAB ${ }^{\circledR}$, LabView ${ }^{\circledR}$ or idustrial SCADAs as SIMATICit ${ }^{\circledR}$ implement an OPC client, and hence can be connected to the plant. Based on the OPC protocol, connectivity of the plant from the WorldWide-Web is also possible (Reyes et al., 2005)

The connectivity of the plant results to be very interesting for the objectives of the plant: education and research. For instance for basic control courses, Simulink or LabView based controllers can be easily implemented and applied to the plant by means of the OPC connection. For more advance courses, an SCADA can be used to design standard controllers or commercial packages such as DMC, for instance. For research, the facilities of the plant allows one to test the controllers easily on the real plant. For instance, in figure 7 it is shown the evolution of the lower levels of the plant in the quadruple tank configuration. The nonminimum phase setting of the plant has been chosen and the plant has been controlled using a multivarible GPC implemented in MATLAB with OPC connection.

\section{CONCLUSIONS}

This paper presents the design and implementation of a laboratory plant based on the quadruple tank process. The main objective of the design of the plant

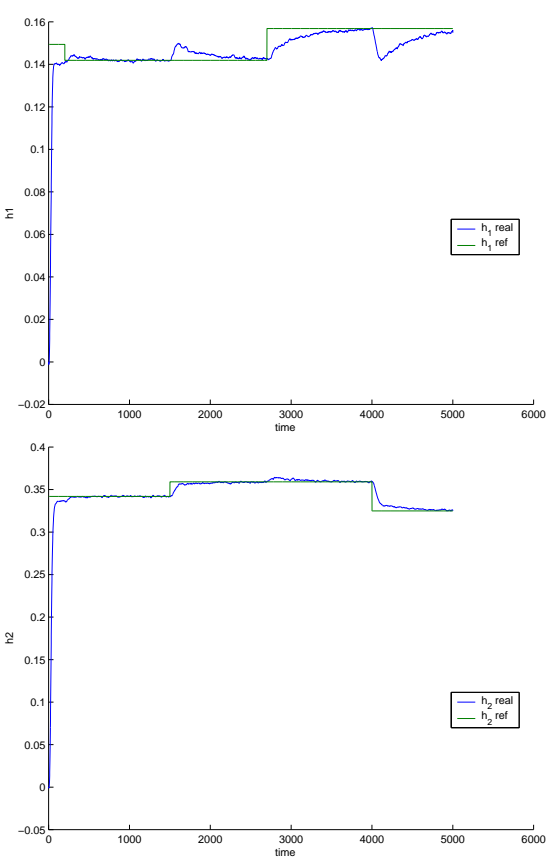

Fig. 7. Evolution of the levels of the non-minimum phase plant controlled by a GPC

is its flexibility. Thus, the plant can be configured to obtain cascaded tanks, a mixture process or a hybrid plant. Moreover the control structure is also flexible allowing one to control in a cascade or in a direct structure by means of the PLC, by using an external device or by means of a computer. In this case, OPC protocol has been chosen to provide connectivity to control applications. In addition, it is interesting to highlight that the plant has been built with industrial instrumentation.

Finally it is worth to remark that this plant has been successfully used for practical works of students in basic control courses as well as our research group.

\section{REFERENCES}

Johansson, Karl Henrik (2000). The quadruple-tank process. IEEE Trans. Automatic Control.

Johansson, K.H., A. Horch, O. Wiljk and A. Hansson (1999). Teaching multivariable control using the quadruple tank process. In: In proceedings of the IEEE Conference on Decision and Control.

Long, C. E., C. E. Holland, and E. P. Gatzke (2005). Experimental air pressure tank systems for process control education. Chemical Engineering Education.

Reyes, C., a. Cepeda, B.Pontes, I. Alvarado and E.F. Camacho (2005). Control de la planta de los cuatro tanques mediante la realización de una pasarela MATLAB-HTTP-OPC. In: Jornadas de Automática.

Rusli, E., S. Ang and R.D. Braatz (2004). A quadruple tank process control experiment. Chemical Engineering Education. 


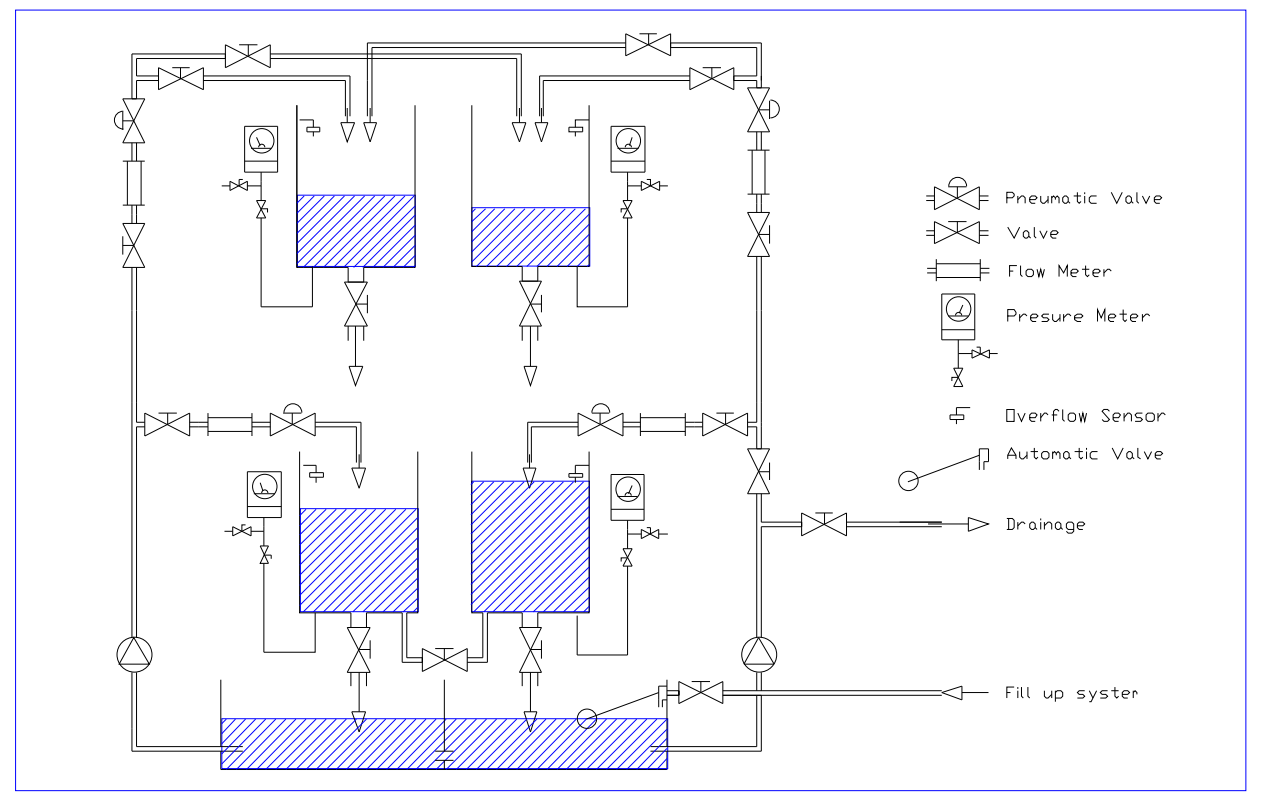

Fig. 8. Laboratory plant layout.

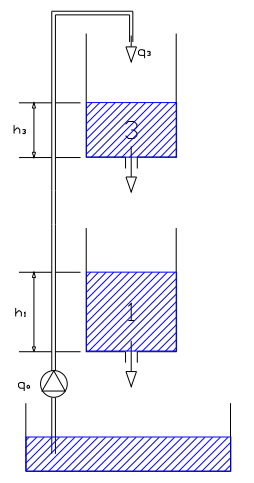

(a) Two cascaded tanks

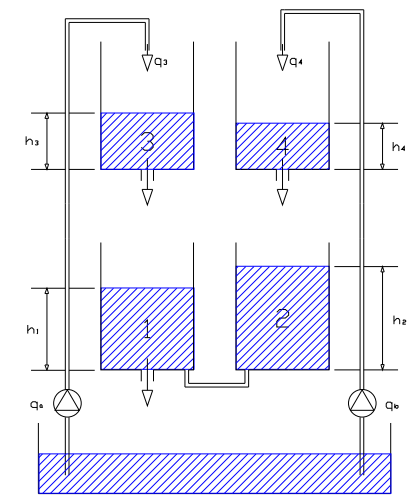

(d) Four coupled tanks.

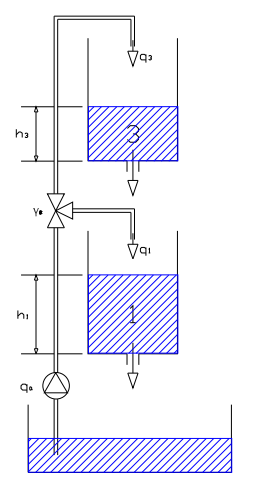

(b) Two cascaded tanks, two inlets.

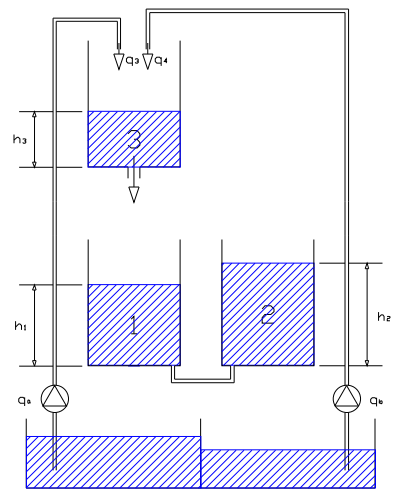

(e) Mixture process.

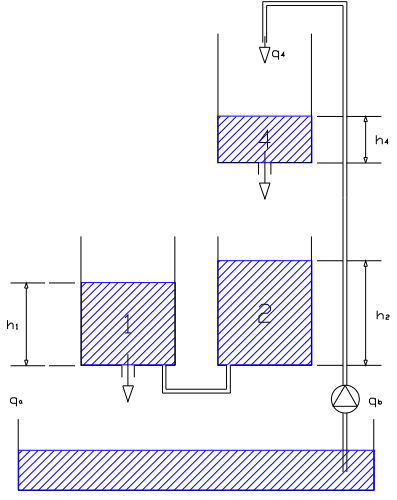

(c) Three cascaded tanks

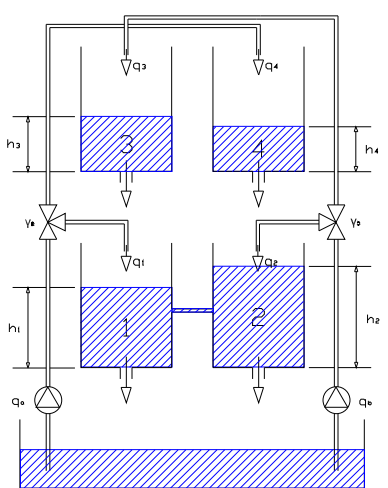

(f) Hybrid process.

Fig. 9. Some configurations of the plant. 